\title{
El enfoque cibernético: La respuesta de profesión contable a los desafios del siglo XXI
}

\section{Introducción}

Como muy bien lo supo expresar la iluminada visión del P. Pierre Teilhard de Chardin, la Materia (i.e. el Mundo) avanza "hacia estados de ordenamiento cada vez más complicados (y en dirección $\multimap$ en el seno- de un "tercer Infinito", el Infinito de Complejidad, tan real como lo Infimo o lo Inmenso). Y la conciencia se presenta experimentalmente como el efecto, o propiedad específica, de esa Complejidad llevada a valores extremos."

"Si se aplica a la historia del Mundo esa ley de recurrencia (denominada "de complejidad-conciencia") se ve cómo se concreta una serie ascendente de puntos críticos y de desarrollos singulares. (...) Aplicado al gran fenómeno de la socialización humana, el criterio de Complejidad-Conciencia aporta las indicaciones decisivas. Por una parte, en la sociedad humana, un irresistible a irreversible ordenamiento técnico-cultural, de dimensiones noosféricas, progresa de modo manifiesto. Y, por otra parte, por un efecto de co-reflexión, el espíritu humano no deja de elevarse colectivamente (gracias a las ligazones tejidas por la técnica) hacia la percepción de dimensiones nuevas..." (Teilhard de Chardin 1973: 91-3).

No se requiere una mente de un gran perfil inquisidor para reconocer en el mundo de hoy las evidencias (o, al menos, los indicios) del desarrollo de una creciente complejificación; esto es, un aumento sucesivo en el número de elementos que intervienen en todos y cada uno de los procesos materiales y humanos, unido a una progresiva frondosidad de sus relaciones.

La complejidad del medio ambiente de los negocios (hábitat natural del Contador Público) es consecuencia de la globalización, los enormes cambios de la economía y la política mundiales, la apremiante necesidad de ser efectivos en el 
racionamiento de recursos escasos, la creciente competencia entre productores que pugnan por ganar una porción mayor del mercado, el empleo intensivo de las llamadas tecnologías de la información y las ingentes cantidades de datos que de estas tecnologías se derivan, entre otros múltiples factores.

Para lidiar con este ambiente complejo, tan difícilmente en una primera aproximación, los directivos de empresas y muy en especial, el Contador Público, deberán aplicarse con gran empeño a "la elaboración de unos ojos cada vez más perfectos" (Teilhard De Chardin 1974: 43) en el seno de un universo que avanza en medio de un rico camino de complejificación creciente.

El objetivo del presente ensayo es tratar de explicar cómo la Contabilidad puede ( $y$ debe) constituirse en la herramienta cibernética fundamental de la cual se deberán valer las organizaciones del futuro basadas en el conocimiento.

\section{La profesión contable rumbo al siglo XXI: Los desafios.}

Desde muy variados flancos y con diversos grados de fuerza, los Contadores (y nuestro ejercicio profesional) estamos sometidos, muy particularmente en los últimos años, a una caterva sucesiva de dramáticos desafíos, que examinaremos en esta sección.

Se ha dicho que "ciertas características antifuncionales, tanto de la teoría como de la práctica de la Contabilidad, conspiran contra la optimización de las empresas, dificultando en lugar de ayudar a mejorar su eficiencia; (...) estos defectos se deben, por lo menos en parte, al retardo cultural relativo de la Contabilidad frente a otras disciplinas, a la resistencia homeostática, a la innovación en un sector profesional de la comunidad empresaria normalmente etnocéntrico, a la falta de base teórica en ciertas áreas de la Contabilidad, esencialmente en materia de costos y valuaciones" (Field y Gabhart 1974: 93)

De otra parte, se ha sostenido que los cuadros directivos han estado influenciados negativamente por la Contabilidad que presenta todas las decisiones como de carácter económico. En tal sentido, el modelo contable estático es un mal pronosticador de la estabilidad de la empresa vista como un sistema, frente a los cambios exigidos por el entorno. Tal como lo sostiene Stafford Beer, la importancia de las mediciones radica no en su valor momentáneo sino en la tasa y en la periodicidad de cambio que expresa. Es necesario, pues, que la Contabilidad entregue información acerca de esas mediciones más que de las mediciones mismas, que resultan meros números (1982: 452).

\subsection{La complejidad: esencia del nuevo entorno empresarial}

Hammer y Champy han señalado que "tres fuerzas, por separado y en combinación, están impulsando a las compañías a penetrar cada vez más profundamente en un territorio que para la mayoría de los ejecutivos y administradores es 
aterradoramente ignoto. Llamamos a estas fuerzas las tres Ces: Clientes, Competencia y Cambio" (Hammer y Champy 1994: 18).

Así, hemos pasado de un mercado de compradores a un mercado de vendedores. Hace algunos años, en plena Era Industrial, la cadena de comercialización se podía graficar como:

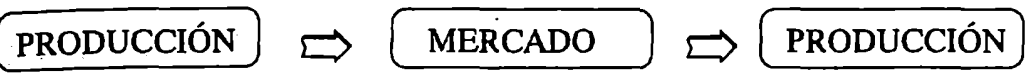

Esto es, que una compañía fabricaba su producto sin tener en cuenta las demandas particulares de sus clientes, simplemente ponía en el mercado su producción y al cabo de un determinado tiempo volvía a poner en marcha la producción y así se iba repitiendo el ciclo.

Hoy, la situación es distinta y se puede graficar más bien así:

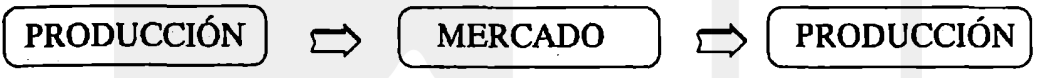

Esto quiere decir que hoy - y de aquí en adelante - las compañías deben estudiar con gran profundidad los gustos de los segmentos de mercado cuyas necesidades quieren satisfacer, luego aplicarse concienzudamente al diseño y fabricación de los productos deseados por los clientes y recién entonces ponerlos en el mercado.

Así, pues, "los que mandan ya no son los vendedores; son los clientes. Hoy, son éstos los que les dicen a los proveedores qué es lo que quieren, cuándo lo quieren y cuánto pagarán. Esta nueva situación está descontrolando a compañías que sólo sabían de la vida en un mercado masivo" (Hammer y Champy 1994: 19).

De otra parte, la competencia se ha incrementado en los últimos años, las compañías no solamente se disputan virtualmente cada cliente sino que usan estrategias competitivas cada vez más sofisticadas. Y se desenvuelven en un ambiente en el cual el cambio se produce más allá de sus expectativas y con una velocidad irrefrenable. Es ya lugar común decir que de aquí al futuro, lo único constante será el cambio.

A estas tres fuerzas (cliente, competencia y cambio) se han unido la amenaza de recursos declinantes, una creciente implantación de la llamada alta tecnología de información y una enormidad de datos disponibles para la toma de decisiones, para acabar de configurar un ambiente complejo. 
La complejidad de un sistema depende, como es sabido, más que de la cantidad de sus elementos constitutivos, de las relaciones que entre estos elementos se establezcan. Fue el filósofo empirista inglés John Locke (1632-1704) quien juiciosamente destacó la importancia de la relación como categoría filosófica, llegando a sostener que el conocimiento correcto de ésta es más valioso que el conocimiento correcto de cuáles son las cosas que se relacionan.

A este respecto, podemos intentar una clasificación de los sistemas valiéndonos del planteamiento de Stafford Beer. El Profesor Stafford Beer, reconocído fundador de la Cibernética Administrativa por los aportes de sus libros "Cybernetics and Management", "Decision and Control", "Heart of Enterprise" y el desarrollo de su "Modelo de Sistema Viable"(Schwaninger 1996: 1) de tan grande interés para los profesionales de la Contabilidad y de la Administración, categorizó a los sistemas en una matriz con dos dimensiones. En una dimensión clasificó a los sistemas en simples, complejos y muy complejos. En la otra dimensión separó a los sistemas en determinísticos y probabilísticos. El foco de la atención de Beer, en sus sucesivos estudios, se centró en los sistemas identificados en la sección de la matriz definida como sistemas probabilísticos y altamente complejos.

La siguiente es una interpretación de dicha clasificación:

Matriz de clasificación de Sistemas

\begin{tabular}{|l|l|l|l|}
\hline \multicolumn{1}{|c|}{ Simples } & \multicolumn{1}{c|}{ Complejos } & Altamente complejos \\
\cline { 2 - 4 } Deterministicos & $\begin{array}{l}\text { Pestillo de ventana } \\
\text { Billar } \\
\text { Distribución de } \\
\text { planta }\end{array}$ & $\begin{array}{l}\text { Computador digital } \\
\text { Sistema planetario } \\
\text { Automatización }\end{array}$ & Vacío \\
\cline { 2 - 4 } & $\begin{array}{l}\text { Tirada de moneda } \\
\text { Control estadístico } \\
\text { de Calidad. }\end{array}$ & $\begin{array}{l}\text { Reflejos condicio- } \\
\text { nados } \\
\text { Probabilidad indus- } \\
\text { trial }\end{array}$ & $\begin{array}{l}\text { La Economía } \\
\text { El Cerebro } \\
\text { LA EMPRESA }\end{array}$ \\
\hline
\end{tabular}

No es muy difícil convenir, después de haber revisado los conceptos precedentes, en que al mundo de la empresa (sistema social abierto) le corresponde la posición matricial $(2,3)$; esto es, el cuadrante de los sistemas altamente complejos y probabilísticos. Qué tan complejo es el sistema "empresa", lo demuestra el hecho de que mientras el número de empleados sube aritméticamente, la cantidad de interacciones posibles entre ellos sube geométricamente. Las relaciones 
interpersonales, en el sistema social que es la empresa, definen una auténtica tela organizacional cuyo tejido se torna más o menos tupido dependiendo del número y fuerza de los enlaces entre las "puntas" del denominado "diamante del sistema de negocios" al que aluden Hammer y Champy en su libro fundacional sobre la "Reingeniería" (1994: 86).

Como se colige fácilmente, esta tela organizacional (al ser la empresa un sistema abierto, de fuerte interacción con el mundo exterior, donde habitan los clientes, los competidores, el gobierno central y sus múltiples instancias reguladoras, los proveedores y otras fuerzas limitantes) está obligada a adaptarse cada vez con mayor rapidez y precisión a los incesantes embates de un ambiente externo que no cesa de topetar.

La empresa, pues, puede ser vista como un sistema complejo adaptivo.

Un sistema adaptivo usa sus recursos para adaptarse a las perturbaciones del entorno interno y externo para sobrevivir y lograr sus objetivos.

Los sistemas adaptivos complejos pueden ser categorizados a su vez como estacionarios y no-estacionarios. Los sistemas estacionarios son aquellos en los cuales las variables cambian en el tiempo, pero la función que las relaciona es constante. Estos sistemas pueden ser representados por modelos cuantitativos mediante el uso de ecuaciones diferenciales y otras técnicas de modelamiento matemático. Se dice que el sistema solar es un sistema estacionario. Las relaciones y los movimientos de los cuerpos en el espacio pueden ser predichos con las leyes de la mecánica orbital (Leyes de Newton). Las funciones que definen estos movimientos no cambian con el tiempo.

Sin embargo, es imposible usar modelos tradicionales de matemáticas o de investigación de operaciones para explicar o modelar sistemas no-estacionarios. Los sistemas con componentes humanos (como lo es una empresa) son conceptualmente no-estacionarios. (Stinson 1996: 3)

El nuevo entomo de los negocios, complejo como hemos dicho, al que tienen que enfrentarse las organizaciones de la llamada Era de la Información queda configurado en gran medida por los supuestos operativos siguientes (Kaplan y Norton 1996: 4-6):

\section{- Funciones Cruzadas}

Las organizaciones de la Era Industrial obtuvieron ventajas competitivas por medio de una alta especialización en habilidades funcionales: en manufactura, compras, distribución, mercadeo y tecnología. Las organizaciones de la Era de la Información operan con procesos integrados que transponen las fronteras organizacionales, combinando los beneficios de la pericia funcional con la velocidad, eficiencia y la calidad de dicha integración. 


\section{- Eslabonamiento de Clientes y Proveedores}

Las organizaciones de la Era de la Información se valen de un racional y efectivo encadenamiento de las relaciones con clientes y proveedores a través de toda la Cadena de Valor para obtener mejoras enormes en costos, calidad y tiempos de respuesta.

\section{- Segmentación de mercados.}

La prosperidad de las empresas de la Era Industrial se nutrió en la oferta de productos y servicios estandarizados y de bajo costo, recogiendo la famosa frase de Henry Ford: "Usted puede escoger el color de carro que prefiera, siempre y cuando sea negro". En la Era de la Información las empresas deberán aprender a ofrecer a sus clientes productos y servicios diseñados tomando en cuenta satisfactores determinados con un gran ajuste a sus necesidades.

\section{- Innovación}

Los ciclos de vida de los productos continúan estrechándose. Las empresas de la Era de Información deberán anticipar los cambios en las preferencias de los consumidores. Aún las empresas que desarrollan productos con ciclos de vida extensos deberán aplicarse a procesos de mejora e innovación continuas, como la única forma de asegurar el éxito en el futuro (esto es: su supervivencia).

\section{- Trabajos Intelectuales}

Las compañías de la Era Industrial crearon claras diferencias entre dos grupos de empleados. La elite intelectual constituida por los gerentes y los ingenieros que usaban sus habilidades analíticas para diseñar productos y procesos, seleccionar y administrar la cartera de clientes y supervisar las operaciones diarias. El segundo grupo estaba compuesto por las personas que directamente fabricaban los productos y suministraban los servicios. Esta fuerza de trabajo directo fue el principal factor de producción de las empresas de la Era Industrial, pero usó solamente sus capacidades físicas no sus habilidades intelectuales, sus mentes. En las postrimerías del siglo XX y el inicio de la Era de la Información, la automatización de procesos industriales ha reducido la necesidad de contar con gente para labores tradicionales y al mismo tiempo la efervescencia de la competencia ha generado una apremiante demanda de gente que realice funciones analíticas. Incluso ahora los trabajadores manuales son valorados por sus sugestiones de mejoras respecto de calidad, reducción de costos y en la disminución de tiempos de ciclo. La administración y explotación del conocimiento de cada uno de los empleados ha empezado a ser un elemento crítico para el éxito de las compañías en la Era de la Información. 


\subsection{La Información: piedra angular de las organizaciones del futuro}

Encaucemos ahora nuestra atención al segundo gran tema de nuestro discurso: la información.

Peter Drucker en su ensayo "La Organización basada en la Información" postula básicamente que "Información significa datos dotados de oportunidad y propósito; de manera que convertir datos en información requiere conocimientos. Y los conocimientos, por definición, son especializados" (1989:200).

A partir de este planteamiento podemos establecer una ecuación emergente:

$$
\text { Información }=\text { datos }+ \text { propósito }+ \text { oportunidad }
$$

Esta ecuación tiene mucho que ver -como lo hemos desarrollado ampliamente en nuestra propuesta al XIV Congreso Nacional de Contadores Públicos del Perú - con la esencia misma de la Contabilidad y el ejercicio profesional del Contador Público.

Desde nuestra perspectiva, el Contador Público es el Administrador de los Flujos Informacionales de las Empresas y son "de su incumbencia, por ejemplo, los computadores que se instalen en las empresas, el diseño de las bases de datos, las tecnologías emergentes para el tratamiento automatizado de las imágenes y toda cuanta herramienta para la gestión de la información nos traiga la modernidad" (Arenas 1996: 19).

La importancia de la información (i.e. el conocimiento) no solamente ha sido reconocida por Drucker como un elemento dinamizador y hegemónico en las organizaciones de los nuevos tiempos sino que también Alvin Toffler ha puesto de manifiesto que las organizaciones de la Tercera Ola habrán de fundarse en un nuevo poder, el Poder del Conocimiento.

Toffler dice que en los años venideros "el control del conocimiento es el punto capital de la lucha mundial por el poder que se entablará en todas y cada una de las instituciones humanas" (1994: 44). Así como el poder se ha desplazado del músculo (es decir, de la fuerza bruta hegemónica en la Era Industrial) hacia el dinero (grandes conglomerados financieros en los años recientes); así, el poder habrá de desplazarse hacia el conocimiento en los próximos años.

Los Contadores Públicos, como muchas organizaciones sociales, hemos prestado muy poco interés al importantísimo tema del poder. Sin embargo, es éste el concepto que amenaza con ser - como lo señala Toffler- uno de los temas que cambiará nuestras vidas en las empresas, y seguramente en la sociedad.

Como se sabe, el "poder es una medida del potencial que tiene una persona para conseguir que los demás hagan lo que ella desea, a la vez que evita verse 
obligada por otros, a hacer lo que no quiere hacer" (Kotter 1982: V) ya que el futuro nos colocará a los Contadores (dueños de la información organizacional) en el ojo de la tormenta, es preciso que nos entrenemos también en los diversos métodos de la obtención del poder.

El poder se obtiene básicamente por tres medios:

1. Logrando el control de los recursos tangibles de la empresa

2. Obteniendo información y control de los medios de información

3. Estableciendo relaciones favorables (creando sentido de obligación, estableciendo una buena reputación profesional, creando sentido de responsabilidad, etc.)

El poder a nivel más general (es decir, el poder político, social, etc.) se ha obtenido además por muchos otros medios. Y nosotros, los Contadores Públicos, parte importante del activo intelectual de las empresas, hemos permanecido - por lo general- ajenos a él. Pero la arena empresarial va a cambiar pronto y a gran velocidad. Es menester prepararnos para el cambio pues la transformación amenaza con ser ruda.

En nuestro caso, el tema del poder en las organizaciones tiene una enorme trascendencia, toda vez que sin un comportamiento orientado al ejercicio del poder y un conocimiento profundo del ejercicio del poder, los esfuerzos de los Contadores Públicos podrían acabar en esfuerzos estériles.

Para alcanzar un estudio de desarrollo profesional superior y, sobre todo, para impulsar a grupos humanos al logro de mejorados objetivos organizacionales, es preciso que el Contador Público conozca los métodos de obtención del poder y los use sin falsos escrúpulos. Insertado en un entomo empresarial basado en la información, es evidente que el Contador Público deberá buscar una posición de poder valiéndose de medios elaborados de obtención de información y control de los medios que ayudan a producirla.

Resumiendo lo tratado aquí diremos que este escenario de organizaciones basadas en la información inscritas y actuantes en un ámbito de complejidad creciente y de lucha por el poder requiere del Contador Público propuestas de genuina coherencia ( y contundencia) conceptual basadas en un campo de visión ampliada (Cf. Arenas 1996) aprovechando herramientas y modelos análogos a los que se usan en la rama científica denominada Cibernética. Este es esencialmente el foco del presente trabajo.

\section{Una Contabilidad para el siglo XXI: Las respuestas}

Frente al enorme desafío de los procesos de complejificación del medio ambiente organizacional y el poder de la información (materia prima de nuestra 
actividad profesional), los Contadores Públicos debemos concentrarnos en la comprensión y el manejo de lo que hemos llamado enfoque cibernético.

Para coadyuvar a un mayor entendimiento del significado y los alcances de este enfoque, en primer lugar, centraremos nuestra atención en lo que podemos definir como el problema de la información para luego pasar revista al concepto de Cibernética y sus consecuencias en la administración de empresas, deteniéndonos en forma puntual en la herramienta cibernética de mayor significación conceptual para los propósitos de nuestra propuesta: el control por retroalimentación o feedback.

\subsection{El problema de la información}

La actuación del Contador Público como Administrador de la Información Organizacional podemos asimilarla al piloto de un avión que tiene que mantener su nave orientada a un objetivo claro (la ciudad de destino) valiéndose de una serie de sensores e indicadores que le van suministrando datos sobre la velocidad del viento, la altitud, el estado de los motores, etc. Sería altamente sospechosos — como lo dicen Kaplan y Norton en su "Balanced Scorecard" —un piloto que solo contara con la ayuda de un único sensor, olvidándose del estado del resto de las variables que podrían afectar la travesía.

Se podrá cuestionar que la responsabilidad final de alcanzar el objetivo no recae en el Contador Público sino en la Gerencia General de las empresas, pero es claro que el Contador es el front-end organizacional respecto a la información. Él es quién deberá captar, analizar, combinar y comunicar la información que ofrezca el mejor ajuste de acciones orientadas al objetivo. En este sentido, el Contador Público resulta ser - - en todo caso- el más capacitado para co-dirigir el rumbo de esa nave llamada empresa.

Hemos dicho, tomando prestado el discurso de Peter Drucker, que información es datos que tienen un propósito en un horizonte de tiempo dado. Bajo esta óptica podemos decir que el problema de la información puede ser visto desde tres perspectivas:

- Las monumentales masas de datos que hoy en día ponen a disposición de las empresas los sofisticados sistemas de computación electrónica que se están desarrollando.

- La variedad de las fuentes desde las cuales es posible extraer datos.

- La necesidad de asimilar estos datos en un horizonte de tiempo normalmente muy reducido.

Se dice que en la llamada Tormenta del Desierto se llegaron a utilizar 3,000 computadoras para proveer de datos al comando de las fuerzas de los Estados Unidos de América. Podríamos usar la analogía que muchos han aplicado y 
decir que el Contador Público es uno de esos generales que tiene que participar en la Guerra por el Mercado que muchos reconocen en nuestros días (Cf. Ries y Trout 1986) aconsejando a los directivos de empresas acudir a la lectura de los manuales de guerra de sun Tzu o del general prusiano Karl von Clausewitz.

Es claro que el Contador en un entorno de combate, enfrentado a enormes masas de datos tienen que cuidarse de verse afectado por aquella patología empresarial tipificada por Drucker como la "computadoritis aguda" (Drucker 1983: 26). No se debe perder de vista que "la información es la versión filtrada de la parte pertinente de aquellos datos en una forma que ayude al decisor a optar entre cursos alternativos de acción" (Stinson 1996: 5). En atención a ello, el Contador Público deberá desarrollar aptitudes y actitudes de discernimiento frente a un enorme hacinamiento de datos, muchos de ellos sino inútiles al menos no pertinentes al logro de los objetivos organizacionales.

Ya hemos dicho que el cambio es una de las fuerzas más poderosas que está golpeando cada vez con renovados bríos el cuerpo orgánico de las empresas. Y como el ambiente cambia, también la forma de ver los acontecimientos debe cambiar. Las organizaciones están requiriendo, hoy más que nunca, nuevos tipos de información. El Contador Público deberá hacer un esfuerzo intelectual importante para idear nuevas formas de integración de información que correspondan a la cantidad y variedad de datos que capta y acopia. $Y$ tener en cuenta que ha habido desde siempre un fuerte énfasis en la recolección y diseminación de datos, sin embargo, la función de procesamiento y análisis que elige lo mejor y transforma los datos relevantes en información no ha recibido el mismo tratamiento.

En los últimos años, se han concentrado los esfuerzos más en las tecnologías de captación de datos que en la transformación y síntesis de esos datos en una forma pertinente a la solución de problemas y logro de objetivos. Como bien lo señalan Franklin White y James Llinas, "la habilidad para emplear múltiples sensores es sólo el resultado de mejoras en tecnologías físicas; sin una correspondiente mejora en la habilidad para procesar efectivamente los datos combinados de sensores, ningún avance en información se producirá" (Franklin White y James Llinas citados en Stinson 1996: 7).

El Contador Público tiene aquí ventaja. Él está capacitado para interpretar las masas de datos que concurren a la empresa, según modelos mentales que ha ido adquiriendo y desarrollando a lo largo de su exposición a ambientes de una gran cantidad de datos provenientes de las más diversas fuentes. Como bien señalan Peters y Waterman, Jr. "el médico experimentado, el artista, el mecánico, todos tienen un rico vocabulario de modelos, que Simon llama ahora los "viejos amigos".

Esta noción explica el verdadero valor de la experiencia en los negocios y ayuda a comprender la importancia de la administración errante. (...) El jefe 
experimentado tiene sus buenos instintos: con su vocabulario de "viejos amigos" puede darse cuenta inmediatamente si las cosas van bien o mal" (Peters y Waterman, Jr. 1982: 68). Deberá responder, mediante la aplicación de modelos mentales de tratamiento de información aprendidos, a la necesidad de hacer más efectivos los datos en busca del logro de los objetivos organizacionales.

La otra perspectiva desde la cual debemos aplicar nuestra visión se refiere a la oportunidad en la entrega de la información. En un ambiente de combate -como se suele describir el actual ámbito de los negocios- es evidente que la provisión rápida de información (es decir, oportuna para una toma de decisiones efectiva) es de vital importancia. El Contador deberá valerse de las modernas tecnologías de la información para poder responder a cabalidad con su objetivo de Administración de la Información Organizacional.

De lo que se trata en esencia es de mantener el Control de la Situación (en el sentido de los académicos rusos), es decir mantener el control y la regulación de los sistemas por medio de la información. Para estos efectos, es necesario que este control situacional se desarrolle dentro del marco referencial del control por realimentación, auténtica piedra angular del enfoque cibernético que pasamos a explicar en la siguiente sección, previo breve vistazo a lo que es la Cibernética.

\subsection{La Cibernética}

Cibernética es un término que se utiliza profusamente hoy en día. Se habla del ciberespacio, los cibernautas, la ciberguerra y de muchos otros ciber. No es muy seguro, sin embargo, que las personas que están usando estos términos tengan una cabal comprensión del significado de la Cibernética.

La Cibernética viene de la palabra griega cubernhihz la cual significa timotel o regulador. Como campo de estudio, la Cibernética fue abordada por primera vez por Ámpere en 1834 en su obra "Essai sur la philosophie des sciences" en la que dio el nombre de "cybernétique" a "la ciencia de las posibles formas de proceder del gobierno" (FRANK s/f: 43). El estudio moderno de la Cibernética empezó en 1948 con la aparición del libro "Cybernetics or Control and Communication in the Animal and the Machine" del matemático estadounidense Norbert Weiner (1894-1964), reconocido como el Padre de la Cibernética moderna, autor además de "The Human Use of Human Beings" (1950).

Escritos de William Ross Ashby (1903-1972), cibernético británico autor de dos libros clásicos como "Design for a Brain" (1952) e "Introduction to Cybernetics" (1956), y Stafford Beer, han ayudado a enriquecer el estudio y la aplicación de los conceptos cibernéticos en las más variadas disciplinas, especialmente en la Administración de Empresas en cuyo campo se ha destacado el último de los nombrados. 
Weiner definió a la Cibernética implícitamente en el título de su libro como la ciencia que estudia el control y la comunicación en el animal y en la máquina. El académico ruso A.N. Kolmogórov en su prefacio a la traducción al ruso del libro de W. Ross Ashby "Introducción a la Cibernética", define el contenido conceptual de esta disciplina científica, en los términos siguientes: "La Cibernética se ocupa de estudiar los sistemas de cualquier naturaleza capaces de percibir, conservar y transformar información y utilizarla para la dirección y la regulación". (Berishmeey y otros 1968: 59). Desde un punto de vista empresarial, de lo que se trata, es de aplicar los mecanismos de control y de comunicación de los seres vivos a las acciones que se desarrollan en una empresa.

Alguna vez hemos escuchado referirse a la Contabilidad como el centro de la inteligencia (i.e. el cerebro) empresarial. Noción que nos parece muy sugerente y rica en matices que se prestan a un desarrollo de inspiración cibernética muy amplio. La Contabilidad y los Contadores Públicos podemos (bajo este concepto sombrilla) aplicarnos con provecho al estudio de los mecanismos usados por el cerebro humano para poder - por analogía - estudiar, diseñar, regular y controlar los procesos de negocios; es decir, convertimos en el auténtico Cerebro Empresarial. Así lo han hecho, en el campo de la computación automatizada, los ingenieros electrónicos y de sistemas que han desarrollado interesantes teorías como las Redes Neurales, los Algoritmos Genéticos, la Inteligencia Artificial y los Ilamados Sistemas Expertos.

Quienes han seguido las huellas intelectuales de Norbert Wiener han llegado a la conclusión de que los conceptos cibernéticos pueden ser aplicables a todos los campos intelectuales.

A propósito, una acotación interesante. Moliere, el gran dramaturgo francés, en su obra "El burgués gentilhombre" nos cuenta la historia del señor Jourdain quien en su pretensión de convertirse en un gentil hombre tomó los servicios de un maestro de música, de un maestro de esgrima, de un maestro de baile y de un maestro de filosofía. Este último en una escena le explica al señor Jourdain que no hay para expresarse más que la prosa o el verso. A lo que Jourdain curioso inquiere: " ¿Cómo? Cuando yo digo: "Nicolasa, tráeme mis pantuflas y dame mi gorro de dormir" ¿eso es prosa?". A lo que el maestro de filosofía responde categórico: "Sï, señor". Y un señor Jourdain sorprendidísimo exclama: “ $\mathrm{A}$ fe mía! Hace más de cuarenta años que hago prosa sin imaginármelo, y os quedo agradecidísimo por habérmelo enseñado". (Moliere 1974: 163). Análogamente, creo que los Contadores nos hemos estado expresando todo el tiempo en términos cibernéticos sin habernos dado cuenta de ello. Prueba al canto: los Contadores Públicos hemos estado haciendo Análisis de Variaciones en Contabilidad de Costos, hemos diseñado Sistema de Control Interno, hemos escrito manuales de procedimientos incluyendo precisas acciones correctivas, hemos hecho ajustes por inflación, presupuestos flexibles, etc. Acciones éstas de neto carácter 
cibernético y todo esto sin habernos dado cuenta, como el Sr. Jourdain. La colaboración de Contabilidad y Cibernética promete - sobre todo para la primera- la configuración de un genuino corpus de conocimiento científico.

Murray Eden ha resumido los conceptos de Norber Wiener señalando que la Cibernética se basa en "la noción de que la información puede ser manipulada como una cantidad matemática; que esta cantidad media entre la transmisión de energía y por lo tanto puede ser usada para controlarla; que la incertidumbre estadística es por sí misma una cantidad relativa a (y degradante de la) información; que la información puede ser usada para guiar la toma de decisiones y clarificar nuestras propias descripciones de un fenómeno del mundo real; que la información podría ser transformada por computación y que el control y la información pueden ser combinados para crear mecanismos complejos que se comporten como si fueran inteligentes..." (Murray Eden citado en Stinson 1996:15).

Otro de los pioneros en el campo de la Cibernética, William Ross Ashby escribió dos de los más importantes libros en este campo: "Introducción a la Cibernética" y "Diseño de un cerebro". En estos libros Ashby trata de determinar la forma en que el cerebro produce el comportamiento intencional, inteligente y adaptivo. Él respondió esto mediante el desarrollo del concepto de ultraestabilidad. La ultraestabilidad es conseguida por un organismo cuando éste se adapta a su medio ambiente manteniendo las variables esenciales que definen el sistema (la interacción del organismo con su entorno) dentro de ciertos límites. Esto es logrado mediante la aplicación de la realimentación en busca del objetivo (goal-seeking feedback).

\subsection{El control por realimentación (feedback)}

La regulación de la temperatura del cuerpo es un ejemplo de un sistema natural de control por realimentación (feedback). Este término involucra la medición de la temperatura actual (o su desviación de aquella considerada ideal) y consecuentemente la estimulación de los procesos de calentamiento o enfriamiento del cuerpo. El objetivo es mantener la tempertura corporal en un nivel óptimo y, en este sentido, el feedback provee los ajustes compensatorios necesarios en las entradas de calor de acuerdo a las mediciones del desempeño (o salidas).

Los sistemas de control por realimentación (feedback) son vitales en cada uno de los aspectos de nuestras vidas. Naturalmente, la ocurrencia de sistemas de realimentación abunda: el caso anteriormente citado, los niveles de azúcar en la sangre y los animales predadores (y presas) son algunos ejemplos.

La construcción de sistemas artificiales de feedback es un producto de la Revolución Industrial. En 1790, Watt inventa el governor (Guilbaud 1956: 11), un aparato cuya función consiste en mantener constante la velocidad de rotación 
de un motor a vapor, no obstante los cambios impredecibles en su carga. Modernos sistemas electrónicos que operan bajos los mismos principios, se usan actualmente para regular la velocidad de las turbinas que generan nuestra energía eléctrica. Sistemas de regulación en los cuales el objetivo es mantener constante algo a despecho de perturbaciones desconocidas e imprevistas son una característica común en modernos sistemas de producción y productos de uso masivo (Farmer 1996: 1).

Ashby estuvo interesado en cómo mantener un sistema en el estado deseado. Es decir, en asegurar que el gobierno de la nave de la que hablaba líneas arriba se pueda mantener apuntando al objetivo. Mediante el siguiente gráfico, tomado de la "Introducción a la Cibernética" de Ashby, se puede explicar aunque de manera muy sucinta, el concepto del control por realimentación, es decir del feedback que es - como hemos dicho y desde nuestra perspectiva personal- el centro neurálgico de la concepción cibernética de la Contabilidad.

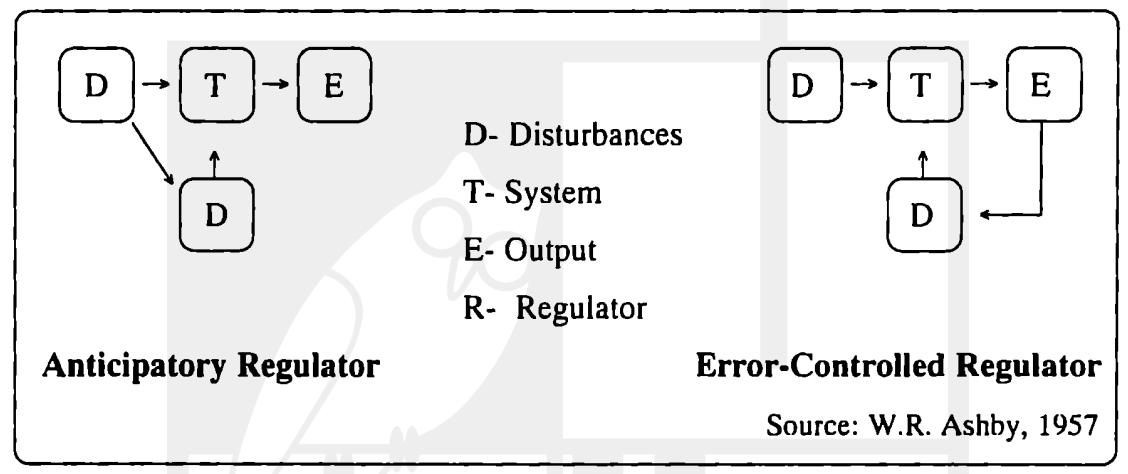

Los gráficos de la figura, representan un sistema $(T)$ sujeto a perturbaciones o turbulencias (D) produciendo salidas definidas por variables esenciales $(E)$ y reguladas por un regulador (R) (Ashby 1960: 303).

Así, el estado del sistema está definido por un conjunto de variables esenciales (E). El sistema (T) sufre una transformación con cada perturbación (D) que actúa sobre el sistema. El objetivo es mantener al sistema dentro de límites predefinidos. El regulador (R) controla las acciones del sistema para responder a cualquier perturbación. El regulador puede recibir realimentación en las formas representadas por los dos diagramas.

En el diagrama de la izquierda (regulador anticipante) el regulador recibe las perturbaciones al mismo tiempo que el sistema. En el segundo (regulador controlado por el error) el regulador recibe información acerca del estado resultante del cambio del sistema. El primer esquema da al regulador la oportunidad de 
reaccionar a los disturbios y evitar impactos pctenciales. El segundo esquema permite solamente una reacción a un estado del sistema ya cambiado.

\section{Contabilidad y Cibernética: Nuestra visión del porvenir.}

Conceptos tales como regulación, control, comunicación, sistema, perturbaciones y objetivo, con los cuales nos hemos estado manejando hasta aquí habrán de llevar al Contador Público a reconocer — cual moderno señor Jourdain- en su práctica diaria y en la esencia de la Contabilidad una genuina sustancia cibernética.

Beer postula que "la nueva ciencia de la Cibernética es la ciencia del control y de la comunicación, dondequiera que éstos se produzcan, en cualquier tipo de sistemas" y que "si la Cibernética es la ciencia del control, la Administración es la profesión del control en cierto tipo de sistemas" (Beer 1982: 247)

Lo que nosotros postulamos ahora, ya a guía de síntesis, respecto del contenido científico de la nueva Contabilidad, es la reformulación de la anteriormente citada definición del profesor Kolmogórov, en los términos siguientes.

La contabilidad se ocupa de estudiar los sistemas de cualquier naturaleza capaces de obtener, conservar y transformar información, y utilizar para el control y la regulación del desarrollo organizacional.

Por otro lado, creemos que si el profesor Beer reconoce a la Administración como la profesión del control, a fortiori debería reconocer más bien a la Contabilidad como la auténtica profesión del control empresarial. A este objetivo, los Contadores Públicos deberíamos aplicar nuestros mejores esfuerzos.

Queremos hacer hincapié que nos estamos refiriendo aquí al control en su sentido menos ortodoxo para los Contadores Públicos, es decir al llamado control homeostático. Esto es, a las acciones que debe emprender el Contador Público para mantener constante, dentro de límites pre-establecidos, determinadas variables consideradas críticas para el logro de los objetivos empresariales.

Así como la homeostasis biológica tiene por finalidad la mejor adaptación (i.e. el "perfeccionamiento") del organismo, igualmente $-y$ por analogía- lo que busca el control contable homeostático, en el sentido que le queremos dar aquí, es la llamada ultraestabilidad del sistema, como la llamó W.R. Ashby. El llamó ultraestabilidad a la capacidad de regresar a una situación direccionada al objetivo después de haber sido perturbado el sistema de un modo no previsto por su creador (Beer 1982: 229) 
En la gráfica siguiente, pretendemos sintetizar gráficamente el concepto de control homeostático que —en nuestra opinión- ejerce el Contador Público y cuyos principales lineamientos hemos tratado de destacar y explicar en las líneas precedentes.

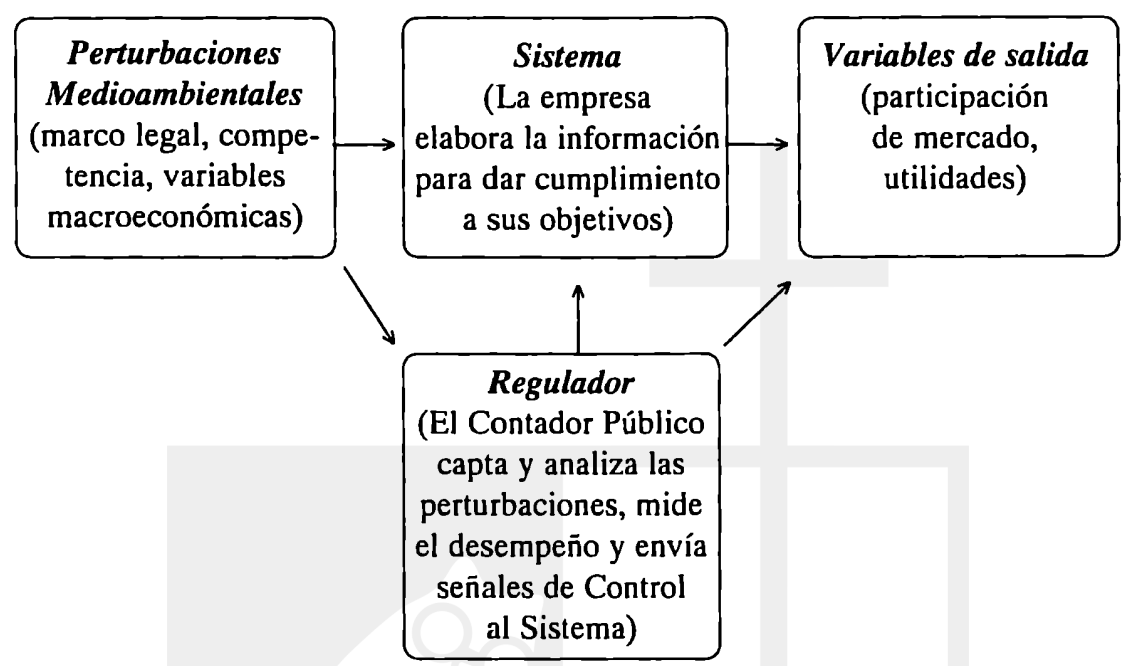

El enfoque cibernético de la Contabilidad ofrece ricas y enormes posibilidades de perfeccionamiento intelectual en el futuro. A partir de un inicial balbuceo del lenguaje cibernético que es fácil entrever en algunos de los mejores trabajos de nuestra especialidad (presupuestos flexibles, informes de retroalimentación, análisis de variaciones, ajustes por variación en el nivel de precios, etc.), los Contadores Públicos iremos ascendiendo hacia estados más elevados de consciencia profesional hasta lograr la consolidación de un estructurado cuerpo científico. Es nuestra más fundada convicción y nuestra más entrañable esperanza.

\section{Guía de discusión}

A fin de delinear un marco conceptual de referencia que nos lleve a un mayor esclarecimiento del enfoque cibernético de la Contabilidad, aquí expuesto, sería interesante buscar respuestas a los planteamientos siguientes:

- ¿Cómo se reconoce la complejidad de un sistema empresarial y cómo se le puede afrontar?

- ¿Cuál es el rol del Contador Público en una empresa basada en la información? 
- ¿Qué enfoque deberá adoptar el Contador Público para conseguir un campo profesional de visión ampliada?

- ¿Cómo se puede reconocer procesos cibernéticos en la Contabilidad?

\section{Conclusiones}

- En los próximos años de la llamada Era de la Información, las empresas se verán enfrentadas a entornos complejos en los cuales el manejo efectivo de la información es de necesidad vital para el gobierno de las empresas.

- Por definición, la Cibernética es la ciencia del control y de la comunicación donde quiera que éstos se produzcan, en cualquier tipo de sistemas. Este concepto se ha aplicado con éxito en diversas disciplinas (Economía, Sociología, Psicología, Ciencias Políticas, etc.). Dicho ejercicio hace sospechar que la aplicación del enfoque cibernético a la Contabilidad deberá devengar iguales o superiores beneficios a nuestro campo profesional.

- El feedback es el control cibernético de los sistemas por medio de la realimentación. Examinados los procesos y lineamientos operativos de la Contabilidad descubrimos que los controles cibernéticos se han aplicado desde muy antiguo en las organizaciones, por intervención de los Contadores Públicos. De esto se infiere que de aquí en más, los Contadores Públicos podemos (y debemos) aplicarnos a una más concreta combinación del enfoque cibernético con los postulados contables para provocar la emergencia de una genuinamente Nueva Contabilidad.

- Dadas las afinidades conceptuales de la Cibernética y la Contabilidad, el enfoque cibernético promete enormes logros en la configuración de un coherente corpus científico para la Contabilidad. Lo cual, a su vez, asegura desarrollos futuros aún insospechados.

\section{Referencias Bibliográficas}

Arenas Herrera, Jesús (1996). 500 años después de Paccioli o la necesidad de una Nueva Cultura Contable. Revista Interamericana de Contabilidad, Abril-Junio, No. 62, Miami, Florida, pp. 11-19.

Ashby, W. Ross (1960) Introducción a la Cibernética. Buenos Aires, Ediciones Nueva Visión.

Beer, Stafford (1982). Decisión y Control. El significado de la investigación de operaciones y la administración cibernética. México, D.F., Fondo de Cultura Económica.

Berishmeey, N. y otros (1968). Introducción a la Cibernética. En Introducción e Historia de la Cibernética, pp. 57-102. México, D.F., Editorial Grijalbo, S.A., Colección 70.

Drucker, Peter F. (1983). El Ejecutivo Eficaz. México, D.F., Editorial Sudamericana.

(1989). Las nuevas realidades. En el Gobierno y la Política... En la Economía y los Negocios... En la Sociedad y en la Perspectiva Mundial. Bogotá, Editorial Norma, S.A. 
Farmer, Peter (1996). Fcedback Control Systems. http://mcmahon.anu.edu.au/technology/ feedback/controlSystems.htm

Field, George A. Y Gabhart, David R.L. (1974) El rctardo cultural relativo de la Contabilidad. Administración de Empresas, IV (47), Bucnos Aircs, 993-1002.

Frank, Helmar (s/f). ¿Qué cs la Cibcrnćtica? En Principios de Cibcrnćtica, pp. 43-57, (Moisés Bendezú), Editora y Distribuidora Lima, S.A., Lima.

George Washington University (s/f) Homepage of William Ross Ashby http:// www.gwu.edu/ asc/biographies/ashby/ashby.htm

Giulbaud, G.T. (1956) La Cibcrnćtica. Barcclona, Vergara Editorial.

Hammer, Michael y Champy, James (1994). Reingeniería. Bogotá, Editorial Norma, S.A.

Kaplan, Robert S. Y Norton, David (1996) The Balanced Scorccard. Translating Strategy into action. Boston, Massachusctts, Estados Unidos de Nortcamćrica, Harvard Business School Press.

Kotter, John P. (1982) El Poder Gerencial. Cómo reconocerlo, obtencrio y usarlo. Mćxico, D.F., Nucva Editorial Intcramcricana, S.A. dc C.V.

Moliére (1974). Tartufo o el Impostor. La Escuela de los Maridos. El burgués (sic) gentilhombre. Buenos Aircs, Editorial Losada, S.A. Traducción de Nydia Lamarque.

Murray, Eden (1983) Cybernctics. En The Study of Information: Interdisciplinary Messages, 427. U. Mansfield. New York, Wilcy \& Sons.

Pelers, Thomas J. Y Waterman, Jr., Robcrt H. (1982) En busca de la excelencia. Expcriencia de las empresas mejor gerenciadas de los Estados Unidos. Bogotá, Editorial Norma.

Ries, Al y Trout, Jack (1986) La Guerra de la Mercadotecnia. México, D.F., Libros McGraw-Hill de México, S.A. de C.V.

Stinson, David R. (1995) Improved Air Campaign Planning through Cybernetics and Situacional Control. Arlington, Virginia, Estados Unidos de Nortcamćrica, Acrospacc Education Foundation - Air Force National Defense Fellows.

Schwaninger, Markus (1996). Stafford Becr http://www.newciv.org/ISSS_Primer/beer.htm Tcilhard de Chardin, Picrre (1973) Yo me explico. Madrid, Taurus Edicioncs. (1974) El fenómeno humano. Madrid, Taurus Ediciones.

Toffler, Alvin (1994) El Cambio del Poder. Barcelona, Plaza \& Janés Editores, S.A. 